\title{
Limits on the primordial stellar multiplicity
}

\author{
S. P. Goodwin ${ }^{1}$ and P. Kroupa ${ }^{2,3}$ \\ ${ }^{1}$ School of Physics \& Astronomy, Cardiff University, 5 The Parade, Cardiff CF24 3YB, UK \\ e-mail: Simon.Goodwin@astro.cf.ac.uk \\ 2 Sternwarte, Universität Bonn, Auf dem Hügel 71, 53121 Bonn, Germany \\ ${ }^{3}$ Rhine Stellar Dynamical Network
}

Received 7 January 2005 / Accepted 10 May 2005

\begin{abstract}
Most stars - especially young stars - are observed to be in multiple systems. Dynamical evolution is unable to pair stars efficiently, which leads to the conclusion that star-forming cores must usually fragment into $\geq 2$ stars. However, the dynamical decay of systems with $\geq 3$ or 4 stars would result in a large single-star population that is not seen in the young stellar population. Additionally, ejections would produce a significant population of close binaries that are not observed. This leads to a strong constraint on star formation theories that cores must typically produce only 2 or 3 stars. This conclusion is in sharp disagreement with the results of currently available numerical simulations that follow the fragmentation of molecular cores and typically predict the formation of 5-10 seeds per core. In addition, open cluster remnants may account for the majority of observed highly hierarchical higher-order multiple systems in the field.
\end{abstract}

Key words. stars: formation - binaries: general - stars: low-mass, brown dwarfs

\section{Introduction}

In the Galactic field most solar-type stars are in multiple systems with a multiplicity fraction, $f_{\text {mult }}=(B+T+Q) /(S+B+$ $T+Q)=0.58$ where $S, B, T, Q$ are the number of single, binary, triple and quadruple systems, respectively (Duquennoy \& Mayor 1991, including significant corrections for incompleteness from the raw value of 0.49). Recent studies have found that $f_{\text {mult }}$ in the field may be somewhat higher than this (Quist \& Lindegren 2000; Söderhjelm 2000). Duquennoy $\&$ Mayor (1991) find the uncorrected ratio of systems to be $S: B: T: Q=1.28: 1: 0.175: 0.05$ (see also Tokovinin \& Smekhov 2002).

There is evidence that $f_{\text {mult }}$ is significantly higher among young stars than in the field (e.g. Leinert et al. 1993; Mathieu 1994; Duchêne 1999; Bodenheimer et al. 2000; Patience et al. 2002; Kroupa et al. 2003; Duchêne et al. 2004; Haisch et al. 2004). In Taurus essentially all stars between 0.3 and $1 M_{\odot}$ are in multiple systems with separations extending to about 1500 au (Leinert et al. 1993; Duchêne et al. 2004; Haisch et al. 2004). Samples of T Tauri stars in other nearby star-forming regions also show that $\mathrm{T}$ Tauri stars generally have a high $f_{\text {mult }}$ and a bias towards larger separations than the main-sequence sample (Duchêne 1999; Patience et al. 2002; Duchêne et al. 2004; Haisch et al. 2004). Such samples are generally biased towards large separations ( $\sim 100$ to $1000-3000$ au) and may miss many close binaries. Generally it is found that $f_{\text {mult }}$ in the sampled separation ranges is approximately twice that in the
Galactic field and if extrapolated to the whole separation range implies $f_{\text {mult }} \sim 1$.

It has been shown that it is not possible to reproduce the observed $f_{\text {mult }}$ through the dynamical evolution of star clusters that are born with a single-star population (Kroupa 1995a). Dynamical interactions are able to disrupt many wide binaries, but are not able to pair stars efficiently or significantly change the properties of close binaries (e.g. Kroupa 1995a,b; Kroupa et al. 1999; Kroupa \& Burkert 2001; Kroupa et al. 2001). This leads us to the conclusion that the majority of stars must form in multiple systems.

All stars form in dense molecular cores (André et al. 2000), and most of these star-forming cores are found within larger complexes that will become star clusters. It is these star-forming cores that must fragment into multiple objects. Recent simulations have shown that cores are able to fragment if there is a small amount of initial rotation (e.g. Burkert \& Bodenheimer 1996), with compression due to an external pressure (e.g. Hennebelle et al. 2003, 2004), or if they contain high- (e.g. Bate et al. 2002, 2003; Delgado Donate et al. 2004) or low- (e.g. Goodwin et al. 2004a,b) levels of turbulence. As reviewed by Duchêne et al. (2004) the models predict the cores to generally fragment into $N=5-10$ pre-stellar objects. But Duchêne et al. point out that this appears to contradict the observational findings according to which no, or very little, higher-order sub-clustering within cores is evident.

In this paper we re-address this problem by considering the elementary constraints on the number of stars that can 
form within a core posed by the decay of higher-order multiple systems. Limits on the number of objects that usually form within cores provide very strong constraints on theories of star formation.

\section{Constraints on the fragmentation of cores}

As a core collapses it is initially isothermal as it is optically thin to its own radiation. At a critical density of $\rho_{\text {crit }} \sim 10^{-13} \mathrm{~g} \mathrm{~cm}^{-3}$ for a $1 M_{\odot}$ core, the gas becomes optically thick and enters an adiabatic regime (e.g. Larson 1969; Tohline 1982; Masunaga $\&$ Inutsuka 2000). This results in a minimum Jeans mass being reached around $\rho_{\text {crit }}$ of $M_{\text {min }} \sim 10^{-2} M_{\odot}$. It is at $\sim \rho_{\text {crit }}$ that fragmentation is expected to occur producing fragments of mass $M_{\min }$. Thus the length scale of the initial multiple systems is expected to be

$R_{\text {form }}<\left(\frac{3 M_{\text {core }}}{4 \pi \rho_{\text {crit }}}\right)^{1 / 3}<125\left(M_{\text {core }} / M_{\odot}\right)^{1 / 3}$ au,

where $M_{\text {core }}$ is the mass of the pre-stellar core. This scenario is supported by the observed peak in the T Tauri binary distribution at 100 au (e.g. Mathieu 1994; Patience et al. 2002).

\subsection{Dynamical decay}

Any (non-hierarchical) multiple system with $N \geq 3$ members is generally unstable to decay with a half-life of $t_{\text {decay }} \sim 80$ crossing times,

$t_{\text {decay }} \sim 14\left(\frac{R}{\mathrm{au}}\right)^{3 / 2}\left(\frac{M_{\mathrm{stars}}}{M_{\odot}}\right)^{-1 / 2} \mathrm{yr}$,

where $R$ is the size of the system and $M_{\text {stars }}$ the mass of the components (Anosova 1986; see also Reipurth \& Clarke 2001). A typical decay time-scale for a system with $R=250$ au $\left(2 R_{\text {form }}\right)$ and $M_{\text {stars }}=1 M_{\odot}$ is then of order $55 \mathrm{kyr}$. The decay of multiple systems has two important consequences: the preferential ejection of the lowest-mass member of the system (Anosova 1986; Sterzik \& Durisen 2003), and the reduction of the semimajor axis of the remaining binary by a factor of 2-4 (Anosova 1986). The reduction in the semi-major axis is significantly greater if ejections occur whilst accretion is on-going (Umbreit et al. 2005). Thus, for decaying higher-order systems $t_{\text {decay }}$ falls rapidly as each ejection reduces the size of the system significantly (as $t_{\text {decay }} \propto R^{3 / 2}$ ). The complete decay of a higher-order system to one binary and a number of single stars is expected to occur in less than $0.1 \mathrm{Myr}$.

This raises the possibility that at least some classical and weak-lined $\mathrm{T}$ Tauri sources have been recently ejected from embedded multiple systems and are coeval with those systems, not older than them. Ejected stars would leave a core in $\sim 100 \mathrm{kyr}$ and depending on the size of a disc that they are able to retain during ejection would appear indistinguishable from classical and weak-lined T Tauri sources. Thus the classical picture that protostellar sources evolve from class $0 \rightarrow I \rightarrow$ $I I \rightarrow$ III may need revision as it does not include (a) the possibility of creating later-type sources immediately through ejections, and (b) the lack of a one-to-one relationship between the numbers of cores and the numbers of later-type sources. In particular, the relative lifetime estimates of young stars assume a one-to-one correlation between embedded and non-embedded sources, the differences in relative numbers being due to the different durations of each phase (e.g. Greene et al. 1994) which may be incorrect.

\subsection{Diluting the multiplicity fraction}

Ejections are predominantly of single stars (see Bate et al. 2002, 2003; Delgado Donate et al. 2004; Goodwin et al. 2004a,b for examples), and therefore ejections dilute $f_{\text {mult }}$ (e.g. Bouvier et al. 2001). A cluster which is (initially) composed of triple systems would have a $f_{\text {mult }}$ of unity, however after the decay of these systems $1 / 3$ of stars will be single, and $2 / 3$ in binaries reducing $f_{\text {mult }}$ to 0.5 . Similarly, a population of quadruples will decay within less than $10^{5}$ yr to a population with $f_{\text {mult }}=1 / 3$, assuming each quadruple decays to two single stars and one binary.

The assumption is made here that the distances between members of the multiple system are approximately equal and that all of the members form at the same time. Simulations of multiple system formation within cores show that often hierarchical systems can form with the members appearing at slightly different times (see Delgado Donate et al. 2004; Goodwin et al. 2004a,b). These multiples remain stable for at least $10 \mathrm{Myr}$ (Delgado Donate et al. 2004). However, higher-order multiples in these simulations generally form as the result of the decay of even higher-order systems ( $N \geq 5$ stars), so that $f_{\text {mult }}$ is low for the whole population.

In contrast to the above expectations from dynamical decay, star-forming regions are typically observed to have an extremely high $f_{\text {mult }}$ (see Sect. 1). In addition to finding a significantly high $f_{\text {mult }}$, Duchêne et al. (2004) find no significant difference in $f_{\text {mult }}$ and the separation distribution between the physically different star-forming regions $\rho$ Oph and Taurus, but a marked difference to the Galactic-field sample. Duchêne et al. (2004; also see Haisch et al. 2004) find that $f_{\text {mult }}$ is significantly higher in young embedded sources $(38 \pm 8 \%)$ than in older flat-spectrum sources $(22 \pm 3 \%)$ in both Taurus and $\rho$ Oph (in both cases this is significantly higher than the field $f_{\text {mult }}$ of $14 \%$ for the same separation range). This suggests that there has been some dynamical decay of young higher-order multiple systems on a time-scale $<1$ Myr. This observation also suggests that some of the late-type sources are single stars ejected from early-type multiple systems.

In a cluster such as the Orion Nebula Cluster, that has an age similar to $\rho$ Oph and Taurus-Aurigae, which has a $f_{\text {mult }} \sim 0.6$ very similar to the field (Duchêne 1999), about one star could have been ejected per binary system, i.e. cores may typically produce unstable triple systems, or alternatively, that roughly $1 / 3$ cores only produced a single star while $2 / 3$ produced binaries. This leads to a basic constraint on star formation within cores in Orion of $1 \leq N \leq 3$. On the other hand, without the need to invoke a different population of cores that form different numbers of objects to other star-forming regions, many (wide) primordial binaries can be destroyed by 
dynamical interactions in such a cluster (Kroupa et al. 2001). It is thus possible to match the Orion and the Galactic field $f_{\text {mult }}$ and separation distribution if the initial population is comprised mainly of binary systems with the T Tauri separation distribution (Kroupa 1995a,b).

It may be that ejected stars have escaped from the cluster (or at least travelled far enough not to be included in samples) and so observations are of preferentially high $f_{\text {mult }}$ regions. However, the ejection velocity of stars is $\sim 1 \mathrm{~km} \mathrm{~s}^{-1}$ (Bate et al. 2002, 2003; Delgado Donate et al. 2004; Goodwin et al. 2004a) which is comparable with the velocity dispersion of star-forming cores. Hence star-forming regions would be expected to be mixed. Also, the potential well of larger clusters (such as Orion) is too deep to allow the loss of stars ejected at such velocities. We conclude that ejected stars and their progenitor systems should normally be coincident.

The generally high $f_{\text {mult }}$ for pre-main sequence late-type stars uncovers an elementary discrepancy between observation and star-formation theory if cloud cores produce $N>3$ stars.

\subsection{Primordial triples}

A non-negligible fraction of $\mathrm{T}$ Tauri stars in the surveyed regions do appear to be hierarchical triples, probably $>25 \%$. Leinert et al. (1993) find 4 higher-order multiples from 45 systems in Taurus, at least 7 more are known with possibly more currently undetected. More recently, Koresko (2002) has used speckle holography at $2 \mu \mathrm{m}$ at Keck to search for tertiary companions among 14 pre-main sequence visual binary systems finding that possibly up to half of the imaged objects may be triples, although clear identification of tertiary companions was possible only in two of the possible 7 cases. Noticeably, a large fraction of Koresko's sample lies in the Ophiuchus cloud, a region that may be seen as a moderately rich cluster, clearly different from the Taurus cloud surveyed by Leinert et al., suggesting that this is a relatively general feature and not a "single cloud" phenomenon. In yet more dispersed star-forming regions (the TW Hya and MBM 12 associations), Brandeker et al. (2003) found a similarly high ratio of triples-to-binaries, though with limited statistical significance.

In the Galactic field, Tokovinin \& Smekhov (2002) suggest that $20-30 \%$ of multiple systems are higher-order hierarchical systems, consistent with the fraction of T Tauri stars suggesting that many of these young systems are stable as found in the simulations of Delgado Donate et al. (2004). The fraction of triple and quadruple systems per star, $\mathcal{F}=(T+Q) /(S+2 B+$ $3 T+4 Q)=0.05$ for the Galactic field, this corresponds to a higher-order multiplicity fraction of $(T+Q) /(B+T+Q)=18 \%$ of all multiple systems.

The very high frequency of primordial binary and triple systems in the Taurus and $\rho$ Oph star-forming regions suggest that very few ejections from multiple systems have occurred. This implies that stars must form in groups of 2 or 3 within cores.

\subsection{Hardening and close binaries}

Further support for our conclusion that $2 \leq N \leq 3$ is provided by the separation distribution of systems. There are many binaries in Taurus with wide separations $>100$ au (roughly double the number found in the field) which also implies that few ejections can have occurred, as ejections rapidly harden binaries so that their semi-major axes are $<10$ au - from an initial separation of 100 au, 2 or 3 ejections are sufficient (Anosova 1986; Umbreit et al. 2005).

Ejections are expected to occur during the main (class 0) accretion phase from basic decay-time arguments (see above), and also as seen in star formation simulations (Bate et al. 2002, 2003; Delgado Donate et al. 2004; Goodwin et al. 2004a,b; see also Reipurth \& Clarke 2001). Ejections during this phase are far more effective at hardening binary systems (see Umbreit et al. 2005). This occurs as ejections harden a lower-mass system than the final system thus requiring less energy. In addition, early hardening tends to lead to a population of equal-mass binaries, as the close low-mass component is more able to accrete high angular momentum material from the disc thus pushing the mass ratio towards unity (Whitworth et al. 1995; Bate \& Bonnell 1997). Such systems are not observed in significant numbers in the field (Duquennoy \& Mayor 1991; Mazeh et al. 1992).

It is not expected that stars would form much closer together than $\sim 20-50$ au (the separation between two minimum Jeans mass objects at $\sim \rho_{\text {crit }}$, see Eq. (1)). Ejections are thought to be the primary method by which these systems are hardened. However, these systems are unlikely to have been hardened during the main accretion phase as they do not strongly favour equal-mass binaries, although short-period binaries do favour more similar component masses than wider binaries (Mazeh et al. 1992). They may however form from the (fairly late) decay of some fraction of the triple systems.

Thus, in order to prevent a large population of close binaries being formed from the decay of unstable triple systems, $N=2$ must be a more common outcome of star formation than $N=3$.

\subsection{Fragmentation consistent with data}

We propose the following picture that is consistent with the data. Of every 100 star forming cores within a cloud roughly 60 will form binary and 40 will form triple systems. Of those which form triples, around 25 are long-lived hierarchical systems, whilst the other 15 triples decay to 15 binaries and 15 single low-mass stars and/or brown dwarfs. The 15 binary systems that result from dynamical decay form the close binary population.

This leads to 115 systems, 15 of which are single stars, 75 are binaries and 25 triples with $f_{\text {mult }}=100 / 115=0.87$ for the whole population. As the ejected stars were the lowest mass members of the triple systems, $f_{\text {mult }}$ remains $\sim 1$ for larger $>0.3-0.5 M_{\odot}$ stars, but is low among low-mass stars and brown dwarfs. Such a primordial population would then either remain essentially unevolved in Taurus-like star-forming regions, or would evolve dynamically in dense Orion-like environments 
on a cluster crossing-time scale (Kroupa 2000; Kroupa et al. 2001, 2003).

\subsection{Brown dwarfs}

The dynamical ejection of sub-stellar embryos has been suggested as a formation mechanism for brown dwarfs (Reipurth 2000; Reipurth \& Clarke 2001). Ejections are expected to occur during the main accretion phase (see above), indeed brown dwarfs must be ejected shortly after they form as the accretion rates of very young sources rapidly grow the embryos to stellar masses (which has been suggested as an explanation of the brown dwarf desert, Reipurth \& Clarke 2003). The ejection of brown dwarfs, despite their low mass, would still have a significant effect on the separation (and hence mass ratios) of young multiple systems if they occur when all of the components have not accreted a significant amount of mass (see also Umbreit et al. 2005). Thus, ejections of brown dwarfs during the main accretion and star formation phase ${ }^{1}$ must be limited in order to avoid creating a large population of close, equal-mass binaries.

The available star-count data in the Taurus star-forming region and in Orion constrains this process to about one brown dwarf being ejected per four stars in both environments (Kroupa \& Bouvier 2003). So this scenario is not ruled-out as a brown dwarf formation mechanism, especially if embryo formation can be delayed and brown dwarfs ejected from systems whose other members have a significantly higher mass, in which case the hardening of the parent system would be reduced. It should be emphasised that, even if all brown dwarfs are ejected embryos, the limit of one brown dwarf per 4 stars (or per two stellar binary systems) still constrains the total number of ejections to be low.

\subsection{Cluster remnants as a source of high-order systems}

As stated above, many of the observed $N=3$ systems in the field may be primordial, i.e. may have formed as stable hierarchical systems from molecular cloud cores. However, some triple and perhaps most higher-order multiple systems may also be formed by star cluster remnants.

de La Fuente Marcos $(1997,1998)$ points out that cluster remnants are characterised by a few binaries, i.e. highly hierarchical high-order multiples, and that the number of such objects can be large given that the majority of Galactic-field stars form in modest clusters. Evaporating clusters decay into highly hierarchical systems of $N \lesssim 15$ members. These remnants will presumably decay further over time possibly producing quadruple or quintuple systems. We can estimate the number of highly hierarchical $N>3$ systems under this scenario as follows:

For a power-law embedded cluster mass function, $\xi_{\mathrm{ecl}}=$ $k M_{\text {ecl }}^{-\beta}$, where $\xi_{\text {ecl }} \mathrm{d} M_{\text {ecl }}$ is the number of embedded clusters with mass in the interval $M_{\mathrm{ecl}}, M_{\mathrm{ecl}}+\mathrm{d} M_{\mathrm{ecl}}$ and $M_{\mathrm{ecl}}$ is the mass in embedded cluster stars, the number of hierarchical

\footnotetext{
1 It is expected that star formation would become difficult after the class 0 phase as most of the gas available for star formation has already been accreted.
}

high-order stellar systems is equal to the number of embedded clusters in a mass interval $M_{\mathrm{ecl}, \min }$ to $M_{\mathrm{ecl}, \max }, N_{\mathrm{ecl}}=$ $\int_{M_{\text {ecl,min }}}^{M_{\text {el } \max }} \xi_{\text {ecl }} \mathrm{d} M_{\text {ecl }}$, since each cluster leaves one such system. The number of stars formed in the embedded cluster population during some epoch is $N_{\mathrm{st}}=\int_{M_{\mathrm{ecl}, \min }}^{M_{\text {ect }}}\left(M_{\mathrm{ecl}} / m_{\mathrm{av}}\right) \xi_{\mathrm{ecl}} \mathrm{d} M_{\mathrm{ecl}}$, where $m_{\mathrm{av}}=0.4 M_{\odot}$ is the average stellar mass which we take to be constant for the present estimate. Thus, for $M_{\mathrm{ecl}, \min }=5 M_{\odot}(\mathrm{a}$ cluster of a dozen stars, Taurus-like object, the least-massive star-forming "unit" known) and $M_{\text {ecl, } \max }=500 M_{\odot}$ (since most Galactic-field stars appear to stem from rather modest clusters, Kroupa 1995a; Adams \& Myers 2001) and for $\beta=2$ (Lada \& Lada 2003) we have $N_{\text {ecl }}=0.19$ and $N_{\text {st }}=4.43$ so that the above defined quantity $\mathcal{F}=N_{\mathrm{ecl}} / N_{\mathrm{st}} \sim 4 \%$. Therefore the number of cluster remnants may be completely sufficient to account for all observed higher-order multiple systems.

\section{Conclusions}

The high multiplicity fraction $f_{\text {mult }}$ of pre-main sequence stars implies that most stars form in multiple systems within dense molecular cores. The decay of young multiple systems generally within $<0.1$ Myr has two main effects: diluting the multiple population through the ejection of predominantly single stars, and hardening the remaining systems.

The observed large fraction of $\sim 10^{6} \mathrm{yr}$ old, wide binary and hierarchical triple systems implies that (a) not much dilution of the binary population can have occurred through ejections, and (b) few ejections can have occurred as systems have not been significantly hardened.

This suggests that most cores form only $2 \leq N \leq 3$ stars. Typical numbers that are consistent with observational constraints are that of 100 birth systems 40 are triple and 60 binaries. Of the 40 triples 25 are long-lived hierarchies while 15 decay to 15 close binaries and 15 single stars. This is then the primordial population as seen dynamically unevolved in Taurus and $\rho$ Oph (Kroupa et al. 2003). The destruction of binary systems by dynamical interactions in dense clusters such as the Orion Nebula cluster further dilutes the binary fraction (Kroupa et al. 2001). Wide and low-mass binaries are preferentially destroyed, and the majority of single low-mass stars can be explained through this process and do not require ejections. Such dynamical disruptions occur in the early stages of cluster evolution and explain the rapid drop in the binary fraction observed between T Tauri stars and older clusters and the Galactic-field main sequence population. The observed variations may be entirely understood through dynamical disruption of wide binary systems in differently crowded regions.

This would imply a remarkable invariance of core fragmentation to differing star-formation conditions. Motte \& Andre (2001) have shown that protostellar cores show different sizes, densities and edge sharpness if they are located in clusterlike environments or sparser associations. Nevertheless, the remarkable success in describing a variety of stellar populations with the same primordial binary population does suggest that core fragmentation may indeed be invariant on the scale of a few hundred au or less. Such an invariance may not be unexpected as, whilst large-scale core properties vary significantly, 
it is only after collapse through several orders of magnitude in density that stars form, and such a collapse may make many large-scale properties irrelevant.

Ejections from cloud cores can lead to brown-dwarfs as ejected embryos, but the frequency of such events is already limited by available data to not more than 1 brown dwarf per two stellar binaries (or one per four stars).

Cluster remnants can form highly hierarchical high-order multiple systems. These contribute to the observed number of $N \geq 3$ stellar systems. Cluster remnants may account for all observed higher-order $(N>3)$ multiple systems. Therefore, possibly only a small fraction of molecular cloud cores have to be able to form long-lived stable hierarchical higher-order multiple systems.

These conclusions place strong constraints on theories of star formation. For any theory of star formation to match observations the majority of cores must fragment into multiple objects. However, they can usually only fragment into 2 or 3 stars. The currently available theoretical results appear to be inconsistent with this, as the cloud-core fragmentation calculations typically form $N=5-10$ fragments per core. This discrepancy may not be unexpected because the theoretical work misses the very essential physics of stellar feedback, and cannot take into account magnetic forces that may play a role in the fragmentation of a core, and simplifies the complex and important thermal physics (in particular simplifying the equation of state of the gas).

Acknowledgements. S.P.G. is supported by a UKAFF Fellowship.

\section{References}

Adams, F. C., \& Myers, P. C. 2001, ApJ, 553, 744

André, P., Ward-Thompson, D., \& Barsony, M. 2000, Protostars and Planets IV, ed. V. Mannings, A. P. Boss, \& S. S. Russell (Tucson: University of Arizona Press), 59

Anosova, J. P. 1986, Ap\&SS, 124, 217

Bate, M. R., \& Bonnell, I. A. 1997, MNRAS, 285, 33

Bate, M. R., Bonnell, I. A., \& Bromm, V. 2002, MNRAS, 332, L65

Bate, M. R., Bonnell, I. A., \& Bromm, V. 2003, MNRAS, 339, 577

Bodenheimer, P., Burkert, A., Klein, R. I., \& Boss, A. P. 2000, in Protostars \& Planets IV, ed. V. Mannings, A. P. Boss, \& S. S. Russell (Tuscon: University of Arizona Press), 675

Bouvier, J., Duchêne, G., Mermilliod, J-C., \& Simon, T. 2001, A\&A, 375,989

Brandeker, A., Jayawardhana, R., \& Najita, J. 2003, AJ, 126, 2009

Burkert, A., \& Bodenheimer, P. 1996, MNRAS, 280, 1190

de La Fuente Marcos, R. 1997, A\&A, 322, 764

de La Fuente Marcos, R. 1998, A\&A, 333, L27
Delgado-Donate, E., Clarke, C. J., \& Bate, M. R. 2004, MNRAS

Duchêne, G. 1999, A\&A, 341, 547

Duchêne, G., Bouvier, J., Bontemps, S., André, P., \& Motte, F. 2004, A\&A, 427, 651

Duquennoy, A., \& Mayor, M. 1991, A\&A, 248, 485

Goodwin, S. P., Whitworth, A. P., \& Ward-Thompson, D. 2004a, A\&A, 414, 633

Goodwin, S. P., Whitworth, A. P., \& Ward-Thompson, D. 2004b, A\&A, 423, 169

Greene, T. P., Wilking, B. A., André, P., Young, E. T., \& Lada, C. J. 1994, ApJ, 434, 614

Haisch, K. E., Jr., Greene, T. P., Barsony, M., \& Stahler, S. W. 2004, AJ, 127, 1747

Hennebelle, P., Whitworth, A. P., Gladwin, P. P., \& André, P. 2003, MNRAS, 340, 870

Hennebelle, P., Whitworth, A. P., Cha, S.-H., \& Goodwin, S. P. 2004, MNRAS, 348, 687

Koresko, C. D. 2002, AJ, 124, 1082

Kroupa, P. 1995a, MNRAS, 277, 1491

Kroupa, P. 1995b, MNRAS, 277, 1507

Kroupa, P. 2000, NewA, 4, 615

Kroupa, P. 2002, Science, 295, 82

Kroupa, P., Petr, M. G., \& McCaughrean, M. 1999, New Astron., 4, 495

Kroupa, P., \& Burkert, A. 2001, ApJ, 555, 945

Kroupa, P., Aarseth, S. A., \& Hurley, J. 2001, MNRAS, 321, 699

Kroupa, P., \& Bouvier, J. 2003, MNRAS, 346, 369

Kroupa, P., Bouvier, J., Duchêne, G., \& Moraux, E. 2003, MNRAS, 346,354

Lada, C. J., \& Lada, E. A. 2003, ARA\&A, 41, 57

Larson, R. B. 1969, MNRAS, 145, 271

Leinert, C., Zinnecker, H., Weitzel, N., et al. 1993, A\&A, 278, 129

Masunaga, H., \& Inutsuka, S. 2000, ApJ, 531, 350

Mathieu, R. D. 1994, ARA\&A, 32, 465

Mazeh, T., Goldberg, D., Duquennoy, A., \& Mayor, M. 1992, ApJ, 401, 265

Motte, F., \& André, P. 2001, A\&A, 365, 440

Patience, J., Ghez, A. M., Reid, I. N., \& Matthews, K. 2002, AJ, 123, 1570

Quist, C. F., \& Lindegren, L. 2000, A\&A, 361, 770

Reipurth, B. 2000, AJ, 120, 3177

Reipurth, B., \& Clarke, C. J. 2001, AJ, 122, 432

Reipurth, B., \& Clarke, C. J. 2003, in Brown Dwarfs, ed. E. Martín (San Francisco: ASP), IAU Symp., 211, 13

Söderhjelm, S. 2000, Astron. Nachr., 321, 165

Sterzik, M. F., \& Durisen, R. H. 2003, A\&A, 400, 1031

Tohline, J. E. 1982, Fundam. Cosmic Physics, 8, 1

Tokovinin, A. A., \& Smekhov, M. G. 2002, A\&A, 382, 118

Umbreit, S., Burkert, A., Henning, T., Mikkola, S., \& Spurzem, R. 2005, ApJ, in press [arXiv: astro-ph/0501075]

Whitworth, A. P., Chapman, S. J., Bhattal, A. S., et al. 1995, MNRAS, 277, 727 